\title{
La réduction du salaire en raison du Covid 19 validée par la Cour Suprême du Brésil
}

Sidnei Machado

\section{(2) OpenEdition}

1 Journals

\section{Édition électronique}

URL : https://journals.openedition.org/rdctss/719

DOI : $10.4000 /$ rdctss. 719

ISSN : 2262-9815

Éditeur

Centre de droit comparé du travail et de la sécurité sociale

\section{Édition imprimée}

Date de publication : 1 avril 2021

Pagination : 142-145

ISSN : 2117-4350

\section{Référence électronique}

Sidnei Machado, "La réduction du salaire en raison du Covid 19 validée par la Cour Suprême du Brésil », Revue de droit comparé du travail et de la sécurité sociale [En ligne], 1 | 2021, mis en ligne le 01 avril 2021, consulté le 03 avril 2022. URL : http://journals.openedition.org/rdctss/719 ; DOI : https:// doi.org/10.4000/rdctss.719

\section{cc)}

Revue de droit comparé du travail et de la sécurité sociale est mise à disposition selon les termes de la Licence Creative Commons Attribution - Pas d'Utilisation Commerciale - Pas de Modification 4.0 International. 


\section{SIDNEI MACHADO}

UNIVERSITÉ FÉdÉRALE DE PARANÁ

\section{LA RÉGLEMENTATION DU TÉLÉTRAVAIL AU BRÉSIL}

Ces derniers mois, le Brésil a traversé une période dramatique au cours de laquelle la crise de la Covid-19 s'est combinée avec une série de crises interconnectées qui ont eu un fort impact sur la réglementation du travail. Le contexte social du pays est marqué par la progression de l'épidémie, bien que le Président Jair Bolsonaro se soit montré déterminé à l'ignorer, et réticent à adopter des mesures strictes pour lutter contre le fléau, notamment en résistant à la vaccination. En 2020, le Brésil est le second pays ayant enregistré le plus grand nombre de décès au monde, après les États-Unis.

La protection du travail est fortement affectée par la crise massive du marché du travail, avec une augmentation brutale du chômage, de l'informalité et de la misère. Cependant, ces indicateurs sociaux négatifs sont également le résultat d'une récession économique pré-pandémique et, dans une large mesure, sont décuplés par la déréglementation généralisée du travail réalisée par la réforme du travail de 2017 (loi n¹3.467) entrée en vigueur le 11 novembre 2017.

Ce scénario impacte profondément la législation du travail au Brésil pour deux raisons.

La première est que la fragilité de la réglementation du travail tend à accentuer les impacts de la crise sociale. La seconde est que les réponses apportées à la crise par le gouvernement de Jair Bolsonaro visent toutes à intensifier les mesures structurelles, pour rendre les règles du travail plus flexibles; la crise s'est avérée être une opportunité d'accroître la déréglementation du travail.

Dans ce contexte national, le présent article analyse la question de la réglementation du télétravail, qui représente une bonne synthèse des tensions ayant surgi, pendant la pandémie, en matière de droit du travail.

\section{I - LA RÉGLEMENTATION DU TÉLÉTRAVAIL}

Au Brésil, le télétravail est une forme émergente de relation de travail, puisqu'il n'était pas encore généralisé avant le début de la pandémie. D’un point de vue réglementaire, ce n'est qu'en 2011 que le travail à distance a fait l'objet d'une première réglementation importante, garantissant l'égalité de statut et de traitement entre les travailleurs à distance et les travailleurs sur place (loi n¹2.551/2011).

Cependant, dans un contexte hostile au droit du travail, c'est la réforme du travail de 2017 (loi n¹3.467/2017) qui a apporté des changements significatifs à la réglementation du temps de travail, en introduisant des garanties contractuelles pour répondre aux demandes formulées par les entreprises en faveur d'une plus grande sécurité juridique dans l'embauche de main-d'œuvre travaillant à distance. 
La réforme de 2017 a représenté un tournant dans la réglementation du télétravail au Brésil par l'introduction d'un nouveau chapitre qui lui est spécifiquement consacré (articles 75-A à 75-E) dans la Consolidation des lois du travail (CLT), par la modification des dispositions relatives au temps de travail (article 62, III), et enfin, en faisant du télétravail l'un des domaines dans lesquels la négociation collective prédomine sur la loi (article 611-A, VIII).

Durant la pandémie, le télétravail a fait l'objet d'une nouvelle réglementation au titre des mesures d'urgence assouplissant les règles issues de la réforme de 2017 pour permettre le recours au télétravail à la seule discrétion et dans l'intérêt de l'entreprise (mesure provisoire $n^{\circ} 927$ du 22 mars 2020).

La régulation du télétravail dans le pays est intervenue avant la pandémie, dans le cadre de la réforme de déréglementation de 2017, puis a été modifiée pendant la période de la pandémie de COVID-19.

Depuis mars 2020, le télétravail est adopté à la discrétion de l'employeur, sans nécessité de négociation collective ou individuelle. Cette conception réglementaire n'a pas créé un obstacle à son adoption d'urgence par les entreprises. Au contraire, elle se développe et s'intensifie pendant la pandémie.

La législation brésilienne issue de la réforme du travail de 2017 définit le télétravail sur la base de deux éléments centraux :

1. le travail est réalisé principalement en dehors des installations de l'employeur ;

2. I'utilisation des technologies de l'information et de la communication pour assumer ce travail. La réglementation brésilienne visant à définir la portée du télétravail ne distingue donc pas le travail à domicile de celui effectué à distance, à domicile ou dans d'autres lieux.

\section{A - CONFLITS D'INTERPRÉTATION}

Les conflits individuels récurrents qui perdurent portent sur des modalités du télétravail, qui font en général l'objet d'actions en justice auprès du Tribunal du travail. Ils sont essentiellement liés à la question de la limitation du temps de travail, qui garantit le droit aux heures supplémentaires, au repos et aux congés.

Malgré la loi de 2011, il existe encore une controverse dans la jurisprudence portant sur la question de savoir si le télétravail doit être qualifié comme une forme de travail externe, sans contrôle des heures de travail et, par conséquent, sans garantir le droit à une limitation des heures de travail légales.

Une autre ligne d'interprétation de la jurisprudence considère qu'il n'y a pas d'incompatibilité entre le télétravail et la comptabilisation des heures de travail, dans la mesure où il est possible d'exercer un contrôle à distance, grâce à la technologie ou par d'autres moyens. Sur la base de ces prémisses interprétatives, il est possible de garantir le paiement des heures supplémentaires au travailleur, même s'il est en télétravail, tant qu'il existe une preuve du contrôle du temps par l'entreprise.

En dépit de quelques nuances dans l'interprétation, la jurisprudence associe le travail à l'extérieur au télétravail lorsqu'il s'agit de statuer sur le contrôle du temps de travail. 
Pour un groupe de juges des tribunaux du travail, le travail à l'extérieur implique la présomption d'une absence de contrôle du temps de travail par l'employeur.

En conséquence, il incombe au salarié de prouver, dans le cadre de la procédure judiciaire, qu'il y a eu un contrôle effectif du temps de travail et, après vérification, il pourra être démontré que le nombre d'heures travaillées est supérieur au nombre d'heures de travail contractuelles, et qu'il a le droit de demander le paiement des heures supplémentaires.

\section{B - POINTS CRITIQUES}

Du point de vue du modèle réglementaire, la réforme contient des points critiques dans ses éléments centraux sur les modalités d'embauche. Un premier problème réside dans l'accent mis sur le contrat individuel en cas d'accord de télétravail.

Le télétravail doit être effectué d'un commun accord et cette prestation de services, dans le cadre du télétravail, doit être expressément incluse dans le contrat de travail individuel sous la forme d'un avenant, qui précisera les activités à réaliser par le travailleur (art. 75-C, CLT).

Le même contrat doit réglementer la fourniture d'équipements et de matériels technologiques. À défaut de clause y relative résultant de la négociation collective, le droit brésilien attribue au contrat de travail individuel la fonction de déterminer diverses conditions générales applicables au télétravail, telles que la santé et la sécurité au travail.

En théorie, il existe une volonté d'adopter cette modalité, avec l'exigence du modèle formel d'un accord individuel entre les deux parties. Cependant, le système brésilien impose des restrictions à la réversibilité du télétravail pour le salarié, dans la mesure où seul l'employeur peut révoquer unilatéralement le télétravail et revenir au présentiel, garantissant dans ce cas une période de transition minimale de 15 jours (article 75-C, paragraphe, CLT).

Ainsi, le retour du salarié au travail en présentiel dépend de la seule volonté de l'employeur, ce qui constitue une grande asymétrie dans la relation contractuelle. En revanche, la même réforme prévoit la possibilité d'une négociation collective spécifique sur le télétravail. C'est l'un des domaines où la convention collective prend le pas sur la loi (article 611-A, VIII, de la CLT).

Le deuxième point est la question de la limitation du temps de travail. La loi brésilienne, avec une volonté de forte déréglementation, établit que le télétravail fait partie des modalités de travail dans lesquelles les salariés ne bénéficient pas d'une limite du nombre d'heures de travail (article 62, paragraphe III, du CLT). Le texte de la réforme de 2017 cherchait à déréglementer le temps de travail pour tenter de neutraliser une jurisprudence qui rejetait la thèse selon laquelle le télétravail n'est pas susceptible d'être contrôlé par l'employeur.

D'une certaine manière, la disposition expresse de la loi de 2017 excluant le régime des heures de travail sert à immuniser la jurisprudence et, en même temps, à créer un effet limitatif sur l'accès à la justice du travail. 
L'absence de liens avec la négociation collective fait du contrat individuel de télétravail un modèle facile à appliquer pour l'employeur. Dans un contexte de grande asymétrie le travailleur est incapable de s'opposer à l'employeur pour faire obstacle au passage au travail à distance ou pour en négocier les conditions.

\section{II - LA RÉGULATION PANDÉMIQUE}

Durant la crise provoquée par la pandémie, l'une des mesures sanitaires les plus largement appliquées a consisté dans le travail à distance, rapidement encouragé et réglementé en tant que mesure d'urgence. La première mesure du travail pour faire face à la crise, la mesure provisoire (MP) 927 du 22 mars 2020, a établi un ensemble de règles pour faciliter le recours au télétravail par les entreprises.

La MP 927 est restée en vigueur jusqu'au 18 juillet 2020, car elle n'a pas été convertie en loi en l'absence de l'approbation du Congrès national. Depuis le 19 juillet dernier, le télétravail est à nouveau régi exclusivement par les normes introduites dans les réformes de 2017.

Cependant, dans l'intervalle de près de cinq mois durant lesquels la MP 927 a été en vigueur, elle a produit des effets juridiques puisque de nombreuses entreprises ont fait un usage intensif de ses règles pour l'adoption du télétravail. La non-approbation de la MP 927 a amené les entreprises à percevoir une insécurité juridique dans l'adoption et le maintien du télétravail.

Le premier impact majeur du terme mis à la MP 927 réside dans le fait que l'adoption du télétravail fait à nouveau l'objet d'un accord entre l'entreprise et le travailleur, et ne relève plus d'une décision unilatérale de l'entreprise, comme le prévoyait la MP 927 qui se contentait d'exiger un préavis d'emploi de 48 heures.

Désormais, l'employeur ne peut pas, à sa seule discrétion, modifier unilatéralement le régime de travail en présentiel pour passer au télétravail, et vice versa. La MP 927 prévoyait, par exemple, que les contacts via des applications de messagerie (WhatsApp et courriel) ne pouvaient pas être considérés comme du temps disponible pour l'employeur.

Face à ce nouveau scénario qui impose une absence de réglementation lors d'une période d'urgence sanitaire dans le pays et, considérant qu'un important contingent de travailleurs reste en télétravail, depuis le 18 juillet 2020, les entreprises et les travailleurs adaptent à nouveau le télétravail aux mécanismes de la loi de 2017, c'est-à-dire par l'intermédiaire de contrats individuels.

\section{A- LES DROITS INDIVIDUELS}

Le travail est si faiblement réglementé dans le pays qu'il existe le risque d'une dilution des droits. Les droits et garanties acquis par le travailleur ont été conçus dans la logique du travail dans l'entreprise, avec un temps de travail défini et une subordination hiérarchique. Le contrat de travail constitue un échange de temps disponible contre un salaire. Ainsi, la limitation du temps de travail se justifie car il s'agit de préserver la santé du travailleur dans le cadre d'un contrat de travail à durée indéterminée. 
Le problème central du télétravail est qu'il a tendance à ne plus permettre de séparer le temps de travail du temps de non-travail, ni le temps de travail du temps de repos. Sans réglementation, c'est un travail sans limitation du nombre d'heures.

D'une part, il a tendance à offrir toutes les garanties de limitation des heures de travail (en théorie, il n'y a pas de contrôle) et à générer du travail sans rémunération des heures supplémentaires. D'autre part, il tend à étendre l'entreprise au domicile, à la voiture, à l'espace privé du travailleur.

Ainsi, tout espace devient espace de travail. Les contrôles, auparavant exercés par les feuilles de présence et la gestion immédiate, sont désormais effectués à l'aide de systèmes d'algorithmes et de données sophistiqués qui mesurent la production en temps réel. Il y a un risque qu'une certaine flexibilité se traduise par une souveraineté sans limite, étant donné que le droit de se déconnecter n'est pas non plus garanti par la loi brésilienne.

La relation entre la santé et le travail est un autre point critique. Dans les locaux de l'entreprise, l'employeur est responsable de la santé et de la sécurité du travailleur. Mais cette prémisse s'étend difficilement au travail à distance, au domicile du travailleur. L'employeur devrait avoir accès au domicile pour vérifier si le lieu est adapté.

Ainsi, il y a une crainte légitime que de nombreux droits dans le contrat de travail puissent être dilués avec le télétravail.

\section{B - LA REPRÉSENTATION COLLECTIVE}

Un grand défi pour le télétravail est la représentation collective. Il s'agit de savoir comment organiser les télétravailleurs éparpillés chez eux ou en coworking, comment les contacter, sans utiliser de moyens à distance, et comment organiser une grève.

Le deuxième point en matière de représentation est le contenu des revendications. Le télétravail conduit à une individualisation renforcée du travail, rendant difficile une possible identification collective, clé de l'action collective. Cela exigera des syndicats qu'ils adoptent de nouvelles stratégies d'action collective.

Dans une autre dimension, le télétravail remet en question un modèle démocratique de relations de travail. Historiquement, les travailleurs ont modelé l'entreprise comme un lieu de travail et le domicile comme un lieu de repos, bien que dans la division genrée du travail, la plupart des femmes poursuivaient leur journée avec des activités relevant de la reproduction sociale. Le travail à domicile était une exception, et comportait des droits égaux. La flexibilité de ce modèle au cours des dernières décennies découle de la négociation collective, comme alternative pour concilier les intérêts, et tenir compte des obligations de soins ou des réformes du travail.

Lors de la crise de la Covid-19, nous avons commencé à disposer de l'option du télétravail sans passer par la représentation collective, laissée à la discrétion de l'entreprise. Cela affaiblit la légitimité démocratique, les représentants des travailleurs n'ayant même pas la possibilité de participer aux délibérations. 


\section{BRÉSIL}

En conclusion, d'un point de vue réglementaire, la réforme du travail de 2017 a mis l'accent sur le modèle contractuel, tout en affaiblissant, par exemple, les règles de limitation du temps de travail.

La réglementation brésilienne est fragile et insuffisante dans la mesure où elle ne donne pas un traitement adéquat à la négociation collective, ne garantit pas le droit à la déconnexion, ne définit pas la façon de contrôler les heures travaillées, et ne prévoit pas de règles de sécurité et la prise en charge des coûts du travail par les entreprises. 